\title{
Análisis factorial de la calidad de vida relacionada con la salud de pacientes que ingresan en una unidad de corta estancia médica
}

\author{
Beatriz Seoane, Fernando de la Iglesia, Ricardo Nicolás, \\ Vicente Ramos, Carlos Pellicer, Fernando Diz-Lois
}

\section{Factor analysis of health related quality of life in patients admitted to a short stay medical unit}

Background: Disease and hospital admission have a great impact on the quality of life of an individual: Aim: To identify the factors associated with health related quality of life (HRQL) in patients admitted to a Short Stay Medical Unit (SSMU). Patients and methods: Prospective cohort study of 335 patients aged 15 to 99 years (196 males) consecutively admitted to the SSMU. In all we recorded sociodemographic data, number of previous hospital admissions, admission type, main diagnosis, lenght of stay, comorbidities, the Goldberg Depression and Anxiety Scale, the Karnofsky Perfomance Scale, the Barthel Index and quality of life with the EuroQol-5D instrument. After studying the associations between variables, we carried out a factor analysis of those that were significantly related to HRQL Results: Mean body mass index was $27.7 \mathrm{Kg} / \mathrm{m}^{2}$ and $83 \%$ were emergency admissions. Heart failure, chronic obstructive pulmonary disease exacerbation, acute coronary syndromes and cardiac arrhythmias, constituted $56 \%$ of all admissions. HRQL was statistically related to age, gender, educational status, caregiving situation, number of previous admissions, main diagnosis, length of stay, Goldberg scale and Karnofsky, Barthel and Charlson indexes. Factor analysis reduced the original variables to five, which explained $67.8 \%$ of the variance, as follows: Factor 1- Karnofsky and Barthel indexes (27.8\%); Factor 2- age, educational status, caregiving situation (12.3\%); Factor 3- the Goldberg scale (10.4\%); Factor 4- admission type (8.8\%); Factor 5- main diagnosis (8.4\%). Conclusions: HRQL in patients admitted to Short Stay Medical Unit is partially related to perfomance status, age, social and psychological status, admission type an the main diagnosis (Rev Méd Chile 2009; 137: 1163-72).

(Key words: Factor analysis, statistical; Karnofsky perfomance status; Quality of life)

Recibido el 19 de diciembre, 2008. Aceptado el 31 de julio, 2009.

Unidad de Corta Estancia Médica, Complexo Hospitalario Universitario A Coruña. A Coruña. España.

\footnotetext{
Correspondencia a: Fernando de la Iglesia. UCEM - $6^{\mathrm{a}}$ planta. Complexo Hospitalario Universitario A Coruña. As Xubias, 86. 15.006 A Coruña. España Tfno: + 34.981.17.80.99. Fax: + 34.981.17.61.97. E mail: Fernando_Iglesia@canalejo.org
} 
Según la definición de la OMS, la salud es un $\checkmark$ estado de bienestar completo, tanto físico como mental y social, y no simplemente la ausencia de enfermedad o incapacidad. El concepto de calidad de vida relacionada con la salud (CVRS) puede derivarse de esta definición multidimensional, agrupando elementos que forman parte del individuo y elementos externos que interaccionan con él y pueden llegar a cambiar su estado de salud ${ }^{1}$. La enfermedad y el ingreso hospitalario provoca un deterioro en la calidad de vida de los pacientes, especialmente con enfermedades cardíacas ${ }^{2}$, si bien algún estudio constata que $81 \%$ de los pacientes ingresados en Medicina Interna mejoran su calidad de vida, aunque sea de forma modesta ${ }^{3}$. Los instrumentos que miden la CVRS son cada vez más utilizados para evaluar procedimientos clínicos, intervenciones terapéuticas y calidad de los cuidados suministrados ${ }^{4}$. Estos instrumentos pueden clasificarse en dos grandes grupos: genéricos y específicos. Los específicos se centran en aspectos propios de una enfermedad o síndrome concreto y tienen la ventaja de ser más sensibles a aspectos de la calidad de vida determinados por efectos de una enfermedad concreta. Los genéricos pueden ser utilizados en distintos tipos de pacientes o poblaciones con la ventaja de que permite comparar el impacto de diferentes enfermedades sobre la CVRS.

La patología que presentan los pacientes atendidos por los internistas afecta a múltiples órganos y sistemas, y en la mayoría de las ocasiones en cada paciente se suman varias patologías. Por ello parece más adecuado aplicar un cuestionario genérico que uno específico para conocer la CVRS de nuestros pacientes. Entre los más conocidos destacan el perfil de salud de Nottingham y el cuestionario de salud SF-365-7. Lo ideal es un cuestionario muy robusto, que se administre de manera rápida y sencilla, y que permita obtener resultados a la vez fiables y válidos. El EuroQol5D (EQ-5D) es un sencillo cuestionario que además de medir la salud en sus dimensiones físicas, psicológicas y sociales, se ha incluido en estudios de coste-efectividad o coste-utilidad y lo hacen útil en la asignación de recursos sanitarios ${ }^{8}$. Desde 1999 contamos con una versión adaptada y validada para su uso en español ${ }^{9}$.

El objetivo del presente estudio es determinar los factores que se relacionan con la CVRS de los pacientes que ingresan en una Unidad de Corta Estancia Médica utilizando como instrumento de medida el EQ-5D y como herramienta en la interpretación de resultados el análisis factorial que posibilita la reducción del número de variables obtenidas ${ }^{10}$.

\section{MATERIAL Y MÉTODO}

El estudio se diseñó como cohorte prospectiva e incluyó a 335 enfermos $(\alpha=0,05 ; \mathrm{d}=5,4 \%)$ que precisaron ingreso en la Unidad de Corta Estancia Médica del Complexo Hospitalario Universitario A Coruña. Este es un hospital terciario universitario de 1.200 camas, 250 de Medicina Interna, que atiende a un área de 500.000 habitantes y que cuenta con una Unidad de Corta Estancia de 40 camas donde ingresan todo tipo de pacientes médicos que a priori tendrán una estancia breve. Anualmente ingresan en ella unos 2.500 pacientes y la media de su estancia es de 4,5 días.

De cada paciente se obtuvieron datos demográficos, nivel de estudios realizados, datos socioeconómicos, lugar de residencia, tipo de convivencia, antecedentes personales y número de ingresos hospitalarios en los 2 años previos. Del ingreso actual se recogió si fue urgente o programado, la duración del mismo y el diagnóstico principal. A los pacientes se le midió su índice de masa corporal en $\mathrm{kg} / \mathrm{m}^{2}$, se valoró su comorbilidad mediante el índice de Charlson ${ }^{11}$, que trata de predecir la mortalidad al año en función de un total de 22 condiciones comórbidas como enfermedad cardíaca, cáncer, SIDA, etc., asignando a cada condición 1, 2, 3 ó 6 puntos dependiendo del riesgo de fallecer asociado a cada una de ellas. Se calculó el nivel de ansiedad y depresión mediante la escala de Goldberg ${ }^{12}$. Esta es una escala de cribado en la que se realizan 4 items iniciales de despistaje en ambas subescalas y un segundo grupo de 5 items en caso de respuestas positivas. Los puntos de corte son $\geq 4$ puntos en la de ansiedad $\mathrm{y} \geq 2$ puntos en la de depresión. También se estableció la dependencia funcional utilizando la escala de Karnofsky y el índice de Barthel $^{13,14}$. El primero es una escala para medir la capacidad del paciente para realizar tareas rutinarias y necesidad de cuidados cuya puntuación oscila entre 0 y 100 de tal manera que cuanto 
más alto mayor capacidad para realizar actividad y menor necesidad de cuidados. El índice de Barthel evalúa el grado de dependencia de los pacientes teniendo en cuenta 10 actividades básicas de la vida diaria puntuando entre 0 y 100. El 100 corresponde con la independencia y se considera dependiente leve si $>60$ puntos, moderado entre 40 y 55 , grave de 20 a 35 y dependiente total $<20$ puntos.

A cada paciente se le realizó el cuestionario de salud EQ-5D el día del alta hospitalaria, refiriendo su estado de salud ese día. Con este cuestionario el propio individuo valora su estado de salud en cinco dimensiones concretas: movilidad, cuidado personal, actividades cotidianas, dolor/malestar y ansiedad/depresión; cada una de estas áreas tiene tres niveles de gravedad: sin problemas, algunos problemas o problemas moderados y problemas graves, puntuándose respectivamente con 1, 2 ó 3 puntos, de tal manera que una mayor puntuación indica mayores problemas en las distintas dimensiones del cuestionario y peor calidad de vida. Presentamos los resultados mediante la agregación de los niveles 2 y 3 para dar dos categorías de pacientes, con y sin problemas en una determinada dimensión. Se obtuvo la puntuación total de EQ-5D mediante la suma de las 5 dimensiones, obteniendo también individuos sin ningún problema (puntuación =5) e individuos con algún problema en alguna dimensión (puntuación $>5$ ). A continuación al paciente se le solicitó una evaluación general de su salud en una escala visual analógica (VAS) vertical de 20 centímetros, milimetrada, que va desde 0 (peor estado de salud imaginable) a 100 (mejor estado de salud imaginable). En ella el individuo marcó el punto en la línea vertical que mejor reflejaba la valoración de su estado de salud global ese mismo día.

Se realizó un estudio descriptivo de todas las variables incluidas con su correspondiente intervalo de confianza del 95\% (IC 95\%). Se estableció la normalidad de las variables en función del resultado del test de Kolmogorov-Smirnov. La consistencia interna del cuestionario EQ-5D se comprobó calculando el coeficiente $\alpha$ de Cronbach en las subescalas y el total del mismo. La asociación entre las subescalas y el total del EQ-5D con las diferentes variables se estudió mediante los test de la t de Student, la U de Mann-Whitney y los coeficientes de correlación de Pearson y Spearman, según procediese. La fuerza de asociación entre dos variables cuantitativas se consideró débil si el coeficiente de Pearson o de Spearman era inferior a 0,25 , moderada hasta 0,50 y fuerte si era mayor de 0,50. A las variables que obtuvieron una correlación estadísticamente significativa se les aplicó una técnica de análisis factorial para reducir o reagrupar el conjunto inicial de variables en otros más pequeños de factores de variables relacionadas. Como método de extracción de factores se empleó el método de componentes principales. Para determinar el número de factores que se extraían se estableció como punto de corte los valores específi$\cos \geq 1,00$. Se realizó rotación de factores con el método varimax para fortalecer el agrupamiento de las variables alrededor del factor asociado. Los registros anotados en la matriz de factores rotados representan los pesos de las variables en relación con cada factor y reciben el nombre de cargas de factor. Al tratarse de una rotación ortogonal, su intervalo oscila entre $-1,00$ y $+1,00$. Se estudió la asociación entre los factores obtenidos y las diferentes áreas del EQ-5D mediante el coeficiente de correlación de Pearson o Spearman, según procediese.

\section{Resultados}

La Tabla 1 recoge las principales características basales de nuestros pacientes. En la Tabla 2 se reflejan los diagnósticos de los pacientes, su estancia, comorbilidad, estatus psicológico y funcional y los resultados de las 5 dimensiones del EQ-5D dicotomizadas. Se analizó la fiabilidad o consistencia interna del cuestionario calculando el coeficiente $\alpha$ de Cronbach, que resultó superior a 0,70 en todas las áreas y alcanzó un valor global de 0,788.

De las variables dicotómicas analizadas sólo se encontraron diferencias significativas en las puntuaciones del cuestionario en cuanto al sexo y al tipo de ingreso. Las mujeres manifestaron peor calidad de vida con menor puntuación en la escala visual $(60,9 \pm 22$ vs $68,2 \pm 21, \mathrm{p}=0,002)$ y peores resultados en las áreas de dolor/malestar, ansiedad/depresión. Asimismo los pacientes que ingresaron de forma programada obtuvieron mejores resultados en todas las dimensiones y el total del EQ-5D y en la VAS $(74,9 \pm 19$ frente a $63,2 \pm 22$ de los que precisaron ingreso urgente, $\mathrm{p}<0,000$ ). 
Tabla 1. Características basales de los pacientes

\begin{tabular}{|c|c|c|c|c|}
\hline & $\operatorname{Media}(d t)$ & Mediana & Rango & $\mathrm{N}(\%)$ \\
\hline Sexo, varón & & & & $196(58,5)$ \\
\hline Edad, años & $67(16)$ & 72 & $15-99$ & \\
\hline $\mathrm{IMC}, \mathrm{kg} / \mathrm{m}^{2}$ & $27,9(4,8)$ & 27,7 & $15,6-54$ & \\
\hline \multicolumn{5}{|l|}{ Lugar de residencia } \\
\hline Urbana & & & & $177(52,8)$ \\
\hline Rural & & & & $158(47,2)$ \\
\hline \multicolumn{5}{|l|}{ Nivel educacional (estudios realizados) } \\
\hline Sin estudios & & & & $41(12,2)$ \\
\hline Primarios incompletos & & & & $128(38,2)$ \\
\hline Primarios completos & & & & $94(28,1)$ \\
\hline Secundarios & & & & $44(13,1)$ \\
\hline Universitarios & & & & $28 \quad(8,4)$ \\
\hline \multicolumn{5}{|l|}{ Estado civil } \\
\hline Soltero & & & & $39(11,6)$ \\
\hline Casado & & & & $207(61,8)$ \\
\hline Separado/divorciado & & & & $11 \quad(3,3)$ \\
\hline Viudo & & & & $78(23,3)$ \\
\hline \multicolumn{5}{|l|}{ Convivencia } \\
\hline Con familia & & & & $292(87,2)$ \\
\hline Sólo & & & & $35(10,4)$ \\
\hline Otra & & & & $8 \quad(2,4)$ \\
\hline \multicolumn{5}{|l|}{ Tipo de ingreso } \\
\hline Urgente & & & & $279(83,3)$ \\
\hline Programado & & & & $56(16,7)$ \\
\hline
\end{tabular}

IMC: índice de masa corporal.

En la Tabla 3 se recoge la asociación entre las diferentes características generales de los pacientes y el cuestionario EQ-5D, tanto las subescalas como el total. El sexo se asoció débilmente con alguna de las áreas del cuestionario. La asociación con la edad fue entre débil y moderada salvo en relación con la dimensión de ansiedad. No se encontró correlación con el IMC del paciente, su lugar de residencia o las personas con las que convivía. Nuestros pacientes presentaron una correlación entre débil y moderada entre el cuestionario y el nivel de estudios, estado civil, número de ingresos hospitalarios en los 2 años previos e ingreso actual urgente o programado.

La correlación entre calidad de vida y diagnóstico ha resultado débil y no con todas las dimensiones, mientras que la estancia y la comorbilidad de los pacientes mostraron un mayor nivel de correlación (Tabla 4). Se obtuvo una fuerte asociación entre el valor total del cuestionario y su subescala de ansiedad con las escalas de ansiedad y depresión de Goldberg. También la correlación es especialmente elevada entre la situación funcional y las dimensiones de movilidad, cuidado personal y actividades cotidianas del EQ-5D (Tabla 4).

El análisis factorial determinó 5 factores que explican $67,9 \%$ de la varianza de los datos (Tabla 5). El factor de mayor peso es la situación funcional donde destacan las variables de los índices de Karnofsky y Barthel. El segundo factor incluye la edad y el estatus social de los pacientes con las variables del nivel de estudios alcanzado y estado civil de los pacientes. El estado desde un punto de vista psicológico de los pacientes consti- 
Tabla 2. Variables de los pacientes y resultado del EQ-5D

\begin{tabular}{|c|c|c|c|c|}
\hline & Media (dt) & Mediana & Rango & (\%) \\
\hline \multicolumn{5}{|l|}{ Diagnósticos } \\
\hline Insuficiencia cardíaca & & & & $52(15,5)$ \\
\hline Arritmia cardíaca & & & & $51(15,2)$ \\
\hline Cardiopatía isquémica & & & & $48(14,3)$ \\
\hline EPOC & & & & $37(11,0)$ \\
\hline Dolor torácico inespecífico & & & & $26 \quad(7,8)$ \\
\hline Infección respiratoria & & & & $22 \quad(6,6)$ \\
\hline Síncope & & & & $13 \quad(3,9)$ \\
\hline ACVA & & & & $11 \quad(3,3)$ \\
\hline Cáncer & & & & $9 \quad(2,7)$ \\
\hline HDA & & & & $9 \quad(2,7)$ \\
\hline Implante/recambio de marcapasos & & & & $3(0-9)$ \\
\hline Otros & & & & $54(16,1)$ \\
\hline Estancia media, días & $4,87 \quad(4,1)$ & 4 & $1-20$ & \\
\hline Índice de Charlson & $1,94 \quad(1,9)$ & 1 & $0-12$ & \\
\hline Escala ansiedad de Goldberg & $2,53 \quad(2,7)$ & 2 & $0-9$ & \\
\hline Escala depresión Goldberg & $2,16 \quad(2,4)$ & 2 & $0-9$ & \\
\hline Índice de Karnofsky & $83,5(17,4)$ & 90 & $40-100$ & \\
\hline Índice de Barthel & $88,8(20,9)$ & 100 & $5-100$ & \\
\hline \multicolumn{5}{|l|}{ EuroQ5D movilidad } \\
\hline & 1 & & & $227(67,8)$ \\
\hline & $>1$ & & & $108(32,2)$ \\
\hline \multicolumn{5}{|l|}{ EuroQ5D cuidado } \\
\hline & 1 & & & $264(78,8)$ \\
\hline & $>1$ & & & $71(21,1)$ \\
\hline \multicolumn{5}{|l|}{ EuroQ5D actividades } \\
\hline & 1 & & & $219(65,4)$ \\
\hline & $>1$ & & & $116(34,6)$ \\
\hline \multicolumn{5}{|l|}{ EuroQ5D dolor } \\
\hline & 1 & & & $218(65,1)$ \\
\hline & $>1$ & & & $117(34,9)$ \\
\hline \multicolumn{5}{|l|}{ EuroQ5D ansiedad } \\
\hline & 1 & & & $186(55,5)$ \\
\hline & $>1$ & & & $149(44,5)$ \\
\hline EuroQ5D VAS & $65,18 \quad(22)$ & 60 & $0-100$ & \\
\hline
\end{tabular}

tuye el tercer factor. Que un ingreso sea programado o urgente es el principal determinante del cuarto factor y el quinto depende del diagnóstico principal que motiva el ingreso de los pacientes.

Los factores obtenidos en el análisis factorial se correlacionan con el EQ-5D en casi todas las áreas, excepto el $4^{\circ}$ y $5^{\circ}$ factor que no se correlacionan (Tabla 6).

\section{DISCUSIÓN}

La cohorte de pacientes de nuestra Unidad incluye a un grupo de pacientes de edad avanzada y con elevada comorbilidad, similar a los pacientes que ingresan en la mayoría de las Unidades de Medicina Interna de los hospitales españoles. 
Tabla 3. Estudio de la correlación entre las puntuaciones del cuestionario de calidad de vida (EQ-5D) y características generales de los pacientes

\begin{tabular}{|lccccccc|}
\hline & Movilid & Cuidado & Activid & Dolor & Ansied & Total & VAS \\
\hline Edad & 0,419 & 0,305 & 0,374 & 0,196 & - & 0,288 & $-0,270$ \\
IMC & - & - & - & - & - & - & - \\
Lugar resid & - & - & - & - & - & - & - \\
Educa & $-0,221$ & $-0,198$ & $-0,181$ & - & - & $-0,190$ & 0,190 \\
Estado civil & 0,210 & 0,179 & 0,188 & 0,157 & - & 0,162 & $-0,194$ \\
Conviv & - & - & - & - & - & - & - \\
Ingres previo & 0,301 & 0,275 & 0,308 & 0,181 & 0,155 & 0,280 & $-0,284$ \\
\hline
\end{tabular}

Movilid: movilidad. Activid: actividades. Ansied: ansiedad VAS: escala visual analógica. IMC: índice de masa corporal. Lugar resid: lugar de residencia. Educa: nivel de estudios Conviv: convivencia. Ingres previo: número de ingresos hospitalarios en los 2 años previos. Tipo ingres: tipo de ingreso Se señalan los valores significativos con $\mathrm{P}<0,01$.

Tabla 4. Estudio de la correlación entre las puntuaciones del cuestionario de calidad de vida (EQ-5D) y el diagnóstico, estancia media y escalas de los pacientes

\begin{tabular}{|lccccccc|}
\hline & Movilid & Cuidado & Activid & Dolor & Ansied & Total & VAS \\
\hline Diagn & $-0,243$ & $-0,154$ & $-0,192$ & - & - & - & - \\
Estanc & 0,304 & 0,303 & 0,281 & 0,215 & - & 0,223 & $-0,237$ \\
Charlson & 0,351 & 0,292 & 0,290 & 0,154 & 0,160 & 0,308 & $-0,253$ \\
AnsGold & 0,180 & 0,226 & 0,242 & 0,225 & 0,581 & 0,437 & $-0,243$ \\
DeprGold & 0,236 & 0,275 & 0,319 & 0,289 & 0,570 & 0,480 & $-0,322$ \\
Karnofsky & $-0,654$ & $-0,623$ & $-0,707$ & $-0,424$ & $-0,212$ & $-0,546$ & 0,418 \\
Barthel & $-0,643$ & $-0,660$ & $-0,633$ & $-0,421$ & $-0,212$ & $-0,447$ & 0,356 \\
\hline
\end{tabular}

Movilid: movilidad. Activid: actividades. Ansied: ansiedad VAS: escala visual analógica. Diagn: diagnóstico. Estanc: estancia media. AnsGold: escala de ansiedad de Goldberg. DeprGold: escala de depresión de Goldberg.

Se señalan los valores significativos con $\mathrm{P}<0,01$.

El análisis de la consistencia interna del cuestionario puso de manifiesto coeficientes de Cronbach altos, ya que si bien para la aplicación clínica lo deseable son cifras de 0,90 ó 0,95, en la comparación de grupos se consideran satisfactorios valores entre 0,7 y $0,8^{15}$.

El presente estudio evidencia 5 factores que se correlacionan con la CVRS percibida por los pacientes que ingresan en una Unidad de Corta Estancia Médica: la situación funcional, la edad y el estatus social, el estatus psicológico, tipo de ingreso y diagnóstico de los pacientes.

Nuestros pacientes mostraron una fuerte asociación entre su situación funcional, objetivada mediante los índices de Karnofsky y Barthel, y su CVRS. Las enfermedades crónicas reducen sustancialmente la calidad de vida y este efecto no difiere de forma importante con el tipo concreto de enfermedad que se padezca ${ }^{16}$. La asociación de comorbilidad física y afectación psicológica 
Tabla 5. Matriz de cargas de factores rotados (método varimax) que explica 67,9 \% de la varianza

\begin{tabular}{|lccccc|}
\hline & \multicolumn{5}{c}{ Factores resultantes del análisis factorial } \\
& 1 & 2 & 3 & 4 & 5 \\
\hline Factor 1 (27,8) Situación funcional & & & & & \\
$\quad$ Karnofsky & $-0,881$ & $-0,264$ & $-0,088$ & 0,134 & $-0,007$ \\
$\quad$ Barthel & $-0,883$ & $-0,214$ & 0,028 & 0,034 & $-0,040$ \\
Factor 2 (12,3) Edad y status social & & & & & \\
$\quad$ Edad & 0,211 & 0,742 & 0,029 & $-0,256$ & $-0,134$ \\
$\quad$ Estudios & $-0,76$ & $-0,668$ & $-0,052$ & 0,198 & 0,033 \\
$\quad$ Estado civil & 0,139 & 0,741 & 0,115 & 0,090 & $-0,026$ \\
Factor 3 (10,5) Situación psicológica & & & & & \\
$\quad$ Ansiedad & 0,058 & $-0,004$ & 0,873 & $-0,064$ & 0,088 \\
$\quad$ Depresión & 0,087 & 0,205 & 0,847 & $-0,038$ & $-0,049$ \\
Factor 4 (8,9) & & & & & \\
$\quad$ Tipo de ingreso & & & & \\
$\quad$ Tipo ingreso & $-0,047$ & $-0,286$ & $-0,106$ & 0,770 & $-0,042$ \\
Factor 5 (8,4) Diagnóstico principal & & & & \\
$\quad$ Diagnóstico & $-0,063$ & $-0,243$ & 0,058 & 0,011 & 0,765 \\
\hline
\end{tabular}

Entre paréntesis figura el porcentaje de la varianza que explica cada factor.

Tabla 6. Estudio de la correlación de la puntuación del cuestionario de calidad de vida (Euro 5Q) y los factores obtenidos en el análisis factorial

\begin{tabular}{|lccccc|}
\hline & \multicolumn{7}{c|}{ Factores obtenidos en el análisis factorial } \\
& 1 & 2 & 3 & 4 & 5 \\
Movilidad & 0,60 & 0,24 & - & - & - \\
Cuidado & 0,56 & 0,18 & - & - & - \\
Actividades & 0,62 & 0,22 & 0,15 & - & - \\
Dolor & 0,36 & 0,15 & 0,22 & - & - \\
Ansiedad & - & - & 0,66 & - & - \\
Total & 0,56 & 0,22 & 0,41 & - & - \\
VAS & $-0,36$ & $-0,22$ & $-0,28$ & - & - \\
\hline
\end{tabular}

Factor 1: Situación funcional. Factor 2: Edad y estatus social. Factor 3: Situación psicológica. Factor 4: Tipo de ingreso. Factor 5: Diagnóstico principal. Se señalan los valores significativos con $\mathrm{P}<0,01$.

deteriora aún más la calidad de vida. Según Keles et al, a medida que aumenta el número de enfermedades crónicas, disminuye paralelamente la calidad de vida y funcionalidad tanto física como mental ${ }^{16}$.
En una cohorte de 226 pacientes mayores de 60 años, cognitivamente intactos con EPOC, insuficiencia cardiaca o cáncer, se demostró que el disconfort físico se asocia con peor salud autoevaluada (OR: 1,9; 95\% IC: 1,2-2,9) y discapacidad 
funcional (OR: 1,8; 95\% IC: 1,2-2,7) ${ }^{17}$. En otro estudio que incluyó a 115 pacientes ambulatorios con cáncer que no recibían tratamiento activo, la presencia de síntomas múltiples y severos multiplica por 4 la probabilidad de peores índices de Karnofsky, y por 3 la probabilidad de obtener peores resultados en las dimensiones cognitiva y social de los test de CVRS ${ }^{18}$. El efecto sinérgico de los síntomas de las distintas enfermedades conduce a una disminución de la situación funcional y de las diferentes dimensiones de la CVRS. Ello explica que en nuestros pacientes su situación funcional sea el principal determinante de su calidad de vida.

Las personas mayores de 75 años tienen significativamente peores resultados en los test de CVRS que las más jóvenes ${ }^{19}$. Nuestros pacientes $>75$ años presentaban un VAS de $60 \pm 22$ frente al $68 \pm 21$ de los $\leq 75$ ( p <0,001). Sin embargo, la mayor edad no siempre se correlaciona con peor calidad de vida. García-Mendoza et al. demuestran en una cohorte de pacientes que comienzan tratamiento renal sustitutivo con hemodiálisis por insuficiencia renal terminal como a los 12 meses del inicio los $\geq 65$ años presentan menos pérdida de su CVRS medida con el cuestionario SF- $36^{20}$. Otro grupo de 753 pacientes japoneses con cardiopatía isquémica y utilizando la versión validada japonesa del SF-36 mostró que la edad se asociaba con menores puntuaciones del componente sumario físico (PCS) y mayores puntuaciones en el componente sumario mental (MCS) ${ }^{21}$

La CVRS percibida por las personas guarda relación directa con su estatus social. En Estados Unidos de Norteamérica las personas con menores estudios y menores ingresos económicos presentan de 10 a 12 puntos menos que la población general utilizando el cuestionario SF-8 ${ }^{22}$. También se objetivan diferencias al comparar género y razas.

La depresión es un conocido factor de riesgo para la rehospitalización de pacientes que precisaron ingreso hospitalario. En el estudio de Kartha et al. multiplica por 3,3 (96\% IC: 1,1-9,3) el riesgo de reingreso en pacientes con hospitalización en los 6 meses previos, controlado por el número de ingresos en el año previo, la comorbilidad medida por el índice de Charlson y el estado funcional físico en base al SF-12 23 . De igual manera los rasgos depresivos se correlacionan con puntuacio- nes significativamente más bajas en los resultados de los test de calidad de vida ${ }^{19}$.

Sentirse deprimido fue el síntoma que más fuertemente se asoció con peor calidad de vida en el estudio de Walke et al., con un OR de 1,9 (95\% IC: $1,35-2,72)^{17}$. Resultados similares obtuvieron Keles et al y por ello concluyen que la detección de la presencia y severidad del distress psicológico asociado al efecto de las enfermedades crónicas y su tratamiento puede mejorar dramáticamente la calidad de vida de estos pacientes $^{16}$. Numerosos estudios demuestran que las personas con sentimientos religiosos o inquietudes espirituales presentan no sólo menos ansiedad, depresión e índices de suicidio, sino también mejor salud en general, incluyendo mayor longevidad, y mayor calidad de vida relaciona con la salud $^{24}$.

Como es de esperar, aquellos de nuestros pacientes que precisaron ingreso de forma urgente, por cuadros agudos o agudización de enfermedades crónicas, y con algunos diagnósticos concretos obtuvieron peores resultados en el test de calidad de vida que los que ingresaron de forma programada y frente a otros diagnósticos. Los test de CVRS han demostrado su validez para añadir información pronóstica en cuanto a la supervivencia de pacientes con infección por VIH, además de los datos clínicos rutinarios como la cifra de CD4 o el estadio de la enfermedad ${ }^{25}$. Además, en concreto el EQ-5D ha demostrado ser un buen predictor tanto de mortalidad como de necesidad de ingreso hospitalario o utilización de los Servicios de Urgencias en pacientes adultos con infección por $\mathrm{VIH}^{26}$

Nuestro estudio presenta limitaciones: la complejidad de los pacientes ingresados en las Unidades de Corta Estancia Médica suelen ser algo menor que la de aquellos que ingresan en Unidades convencionales, los hallazgos podrían ser diferentes si el cuestionario de calidad se realiza el día del ingreso o durante el mismo y no el día del alta, y los resultados de este trabajo pueden no ser extrapolables a otros entornos sanitarios diferentes de otros países.

En conclusión, la CVRS percibida por los pacientes que ingresan en una Unidad de Corta Estancia Médica está parcialmente relacionada con su situación funcional, su edad y estatus social, su estatus psicológico, tipo de ingreso y diagnóstico 
principal. Sin embargo, estos factores solos no pueden predecir la amplia variabilidad de la calidad de vida medida por el cuestionario, y la contribución de cada uno de estos factores es limitada. Nuestro trabajo apoya la realización de test de CVRS robustos, de administración sencilla

\section{REFERENCIAS}

1. Casa Anguita J, Repullo labrador JR, Pereira Candal J. Medidas de calidad de vida relacionada con la salud. Conceptos básicos, construcción y adaptación cultural. Med Clin (Barc) 2001; 116: 26-33.

2. Spiraki C, Kaitelidou D, Papakonstantinou V, PrezeRakos P, Maniadakis N. Health-related quality of life measurement in patients admitted with coronary heart disease and heart failure to a cardiology department of a secondary urban hospital in Greece. Hellenic J Cardiol 2008; 49: 241-7.

3. Eriksen BO, Kristiansen IS, Nord E, Pape JF, Almdahl SM, Hensrud a et al. Does admission to a department of internal medicine improve patients' quality of life? J Intern Med 1998; 244: 397-404.

4. Guyatt GH, Feeny DH, Patrick DL. Measuring healthrelated quality of life. Ann Intern Med 1993; 118: 622-9.

5. Alonso J, Prieto L, Antó JM. The Spanish version of the Nottingham Health Profile: a review of adaptation and instrument characteristics. Qual Life Res 1994; 3: 385-93.

6. Ware JE Jr, Sherbourne CD. The MOS 36-Item Short Form Health Survey (SF-36). Med Care 1992; 30: 473-82.

7. Alonso J, Prieto L, Antó JM. La versión española del SF-36 Health Survey (Cuestionario de Salud SF-36): un instrumento para la medida de los resultados clínicos. Med Clin (Barc) 1995; 104: 771-6.

8. Brooks R. EuroQol: the current state of play. Health Policy 1996; 37: 53-72.

9. Badia X, Roset M, Montserrat S, Herdman M, Segura A. La versión española del EuroQol: descripción y aplicaciones. Med Clin (Barc) 1999; 112 (Supl 1): 79-86.

10. De la Iglesia Martínez F, De la Fuente Cid R, Ramos Polledo V, Pellicer Vázquez C, Nicolás Miguel R, DizLois Martínez F. Análisis factorial de la calidad de vida relacionada con la salud de pacientes con enfermedad pulmonar y obstructiva crónica. Arch Bronconeumol 2001; 37: 410-6.

11. Charlson Me, Pompei P, Ales KL, Mackenzie CR. A new method of classifying prognostic comorbidity in y rápida, porque aporta información adicional a las variables ya conocidas, y consideramos interesante realizar nuevos estudios que los valoren como herramienta para predecir mortalidad y reingreso hospitalario en pacientes médicos.

longitudinal studies: development and validation. J Chronic Dis 1987; 40: 373-83.

12. De la Gándara J. Manejo de depresión y ansiedad en atención primaria. Aten Primaria 1997; 20: 389-94.

13. Sitjas Molina E, San José Laporte A, Armadans Gil L, Mundet Tuduri X, Vilardell Tarrés M. Factores predictores del deterioro funcional geriátrico. Aten Primaria 2003 ; 32: 282-7.

14. Martínez JM, Riera Be, Ibarbia CG, Días de LamaDRID CV, MACías JG. Características funcionales y cognitivas de los ancianos ingresados en un Servicio de Medicina Interna. Rev Clin Esp 2006; 206: 188-90.

15. Bland JM, Douglas G. Statistics notes: Cronbach's alpha. BMJ 1997; 314: 572.

16. Keles H, Ekici A, Ekici M, Bulcun E, Altinkaya V. Effect of chronic diseases and associated psychological distress on health-related quality of life. Intern Med J 2007; 6-11.

17. Walke LM, Byers Al, Gallo WT, Endrass J, Fried TR. The association of symptoms with health outcomes in chronically ill adults. J Pain Symptom Manage 2007; 33: 58-66.

18. Ferreira KA, Kimura M, Teixeira MJ, Mendoza TR, Da Nóbrega JCM, Graziani SR, TAKagaki TY. Impact of cancer-related symptom synergisms on health-related quality of life and performance status. J Pain Symptom Manage 2008; 35: 604-16.

19. Kanauchi M, Kubo A, Kanauchi K, Saito Y. Frailty, health-related quality of life and mental well-being in older adults with cardiometabolic risk factors. Int J Clin Pract 2008; 62: 1447-51.

20. García-Mendoza M, Valdés C, Ortega T, Rebollo P, Ortega F. Differences in health-related quality of life between elderly and younger patients on hemodialysis. J Nephrol 2006; 19: 808-18.

21. Nishiyama S, Momomura S, Ishiwata S, Daida H, Hara K, Nishimura $S$ et al. Health-related quality of life in Japanese patients with ischemic heart disease: a multicenter cooperative investigation assessed using SF-36. J Cardiol 2005; 46: 211-20.

22. Lefante JJ Jr, Harmon GN, Sabih KM, Barnard D, Webier LS. Use of the SF-8 to assess health-related quality of life for a chronically ill, low-income population partipating in the Central Louisiana 
Medication Access Program (CMAP). Qual Life Res 2005; 14: 665-73.

23. Kartha A, Anthony D, Manase CS, Greenwald JL, Chetty VK, Burgués JF et al. Depresión is a risk factor for rehospitalization in medical inpatients. Prim Care Companion J Clin Psychiatry 2007; 9: 256-62.

24. Mueller PS, Plevak DJ, Rummans TA. Religious involvement, spirituality, and medicine: implications for clinical practice. Mayo Clin Proc 2001; 76: 1225-35.
25. Cunningham We, Crystal S, Bozzette S, Hays RD. The association of health-related quality of life with survival among persons with HIV infection in the United Status. J Gen Intern Med 2005; 20: 21-7.

26. Mathews WC, May S. EuroQol (EQ-5D) measure of quality of life predicts mortality, emergency department utilization, and hospital discharge rates in HIVinfected adults under care. Health Qual Life Outcomes 2007; 5: 5. 